\title{
State selective electron injection in non-aggregated titanium phthalocyanine sensitised nanocrystalline $\mathrm{TiO}_{2}$ films $\uparrow$
}

\author{
Emilio Palomares, ${ }^{a, c}$ M. Victoria Martínez-Díaz, ${ }^{b}$ Saif A. Haque, ${ }^{a}$ Tomás Torres*b and \\ James R. Durrant*a \\ a Department of Chemistry, Imperial College London, South Kensington, London, UK SW7 $2 A Y$. \\ E-mail: j.durrant@imperial.ac.uk; Fax: +44 207594 5801; Tel: +44 2075945321 \\ b Departamento de Química Orgánica (C-I), Universidad Autónoma de Madrid, 28049-Madrid, Spain. \\ E-mail: tomas.torres@uam.es \\ c Institut de Ciencia Molecular (IcMol), Universitat de Valencia, 46100-Burjassot, Valencia, Spain
}

Received (in Cambridge, UK) 27th May 2004, Accepted 5th July 2004

First published as an Advance Article on the web 3rd August 2004

We describe a novel titanium phthalocyanine that shows no aggregation when anchored to nanocrystalline $\mathrm{TiO}_{2}$ films through its axial carboxylated ligand without the use of coadsorbents; state selective electron injection into the $\mathrm{TiO}_{2}$ is demonstrated, resulting in efficient photocurrent generation in dye sensitised photoelectrochemical solar cells.

The dye sensitisation of wide-band gap nanocrystalline semiconductors is attracting renewed interest due to the development of efficient photoelectrochemical solar cells ${ }^{1}$ based on ruthenium bipyridyl dyes. The efficiency of these devices is, however, significantly limited by the low optical absorbance of such dyes in the red/near infrared spectral regions. Hence, extensive research efforts are targeting new sensitiser dyes with stronger absorbance over this spectral region.

Phthalocyanines $(\mathrm{Pc})$ are well known chromophores for their intense absorption in the UV/blue (Soret band) and the red/near IR ( $\mathrm{Q}$ band) spectral regions, as well as for their electrochemical, photochemical and thermal stability. They are therefore attractive as sensitiser dyes for dye sensitised solar cells (DSSC's), ${ }^{2}$ as well as other photovoltaic devices. ${ }^{3}$ However, the applications of Pc dyes in DSSC's to date have been limited due to problems associated with aggregation of the dye on the metal oxide surface. Moreover, phthalocyanines suitable for DSSC's typically have a poor solubility in organic solvents, and a strong tendency to aggregate on the film surface, resulting in rapid deactivation of the dye excited state and low sensitising efficiency. Several groups ${ }^{2,4}$ have studied the co-adsorption of small organic acids with the Pc sensitiser to minimise the aggregation process, resulting in a limited improvement in device performance.

Here we report the synthesis of a new titanium phthalocyanine (TiPc) and its application to the sensitisation of nanocrystalline, mesoporous $\mathrm{TiO}_{2}$ films. The choice of Ti as a central metal allows axial ligation to the metal center, as reported previously, ${ }^{5,6}$ the axial ligand being employed to anchor the dye to the nanocrystalline particles and to prevent the aggregation of the macrocycles. On the other hand, the bulky peripheral tert-butyl substituents help to solubilise the dye in common organic solvents and to avoid dye aggregation. Using this axial ligation approach we are, moreover, able to control the distance between the HOMO orbital of the chromophore and the $\mathrm{TiO}_{2}$ surface and thereby the electron injection and recombination dynamics.

Titanium(Iv) tetra(tert-butyl)phthalocyanine (TiPc) was synthesised according to a literature procedure and characterised by ${ }^{1} \mathrm{H}$ NMR and UV-Vis spectroscopies, MALDI-TOF spectrometry and elemental analysis.

The compound TiPc showed electrochemical stability, with reversible dye oxidation being observed with a mid-point potential of $0.950 \mathrm{~V}$ vs. $\mathrm{Ag} / \mathrm{AgCl}$. The solution absorption spectrum showed

$\dagger$ Electronic supplementary information (ESI) available: calculated LUMO and HOMO orbitals, excitation and emission spectra, cyclic voltammetry and experimental details. See http://www.rsc.org/suppdata/cc/b4/b407860h/ the expected intense Q-band absorption transition $\left(\lambda_{\max }=702 \mathrm{~nm}\right.$, $\left.\varepsilon=135000 \mathrm{dm}^{3} \mathrm{~mol}^{-1} \mathrm{~cm}^{-1}\right)$. ZINDO/1 semi-empirical calculations indicated that both the HOMO and LUMO orbitals are delocalised over the conjugated macrocycle, with only minimal contributions from either the central metal or axial ligand. Full synthetic details and characterisation of the compound are given in the supplementary information.

The dye showed strong adsorption to nanocrystalline $\mathrm{TiO}_{2}$ electrodes, as illustrated from the $\mathrm{TiPc} / \mathrm{TiO}_{2}$ film spectrum shown in Fig. 1. From the magnitude of the $\mathrm{TiPc} / \mathrm{TiO}_{2}$ Q-band optical absorbance, we estimate a saturated dye loading of 31 nanomoles per $\mathrm{cm}^{2}$ for the $4 \mathrm{~mm} \mathrm{TiO}$, consistent with monolayer surface coverage assuming a dye area of $290 \AA^{2}$.

The Q-band optical absorbance of the $\mathrm{TiPc} / \mathrm{TiO}_{2}$ film exhibits a small broadening relative to the solution spectrum, typical of most adsorbed sensitiser dyes, but no change in structure or peak position was observed, therefore, indicating minimal dye aggregation. The absence of significant dye aggregation is further supported by transient emission studies of the dye adsorbed to control nanocrystalline $\mathrm{ZrO}_{2}$ films, as shown in Fig. 2. The high conduction band edge of $\mathrm{ZrO}_{2}$ precludes electron injection from the dye excited state. The $\mathrm{TiPc} / \mathrm{ZrO}_{2}$ exhibits an intense, near monoexponential fluorescence decay $(\tau \sim 1.6 \mathrm{~ns})$ indicative of negligible dye aggregation.

As illustrated in Fig. 2, strong quenching of the TiPc emission was observed for the $\mathrm{TiPc} / \mathrm{TiO}_{2}$ films assigned to electron injection from the excited state of the phthalocyanine into the conduction band of the $\mathrm{TiO}_{2}$. The occurrence of efficient electron injection was further confirmed by transient absorption studies as shown in Fig. 3. Following excitation at $450 \mathrm{~nm}$, a long-lived absorption transient is observed, assigned to the photoinduced absorption of the $\mathrm{TiPc}^{+} / \mathrm{TiO}_{2}\left(\mathrm{e}^{-}\right)$state. This transient signal exhibits slow decay dynamics, assigned to interfacial charge recombination with a half lifetime of $\sim 0.01 \mathrm{~s}$.

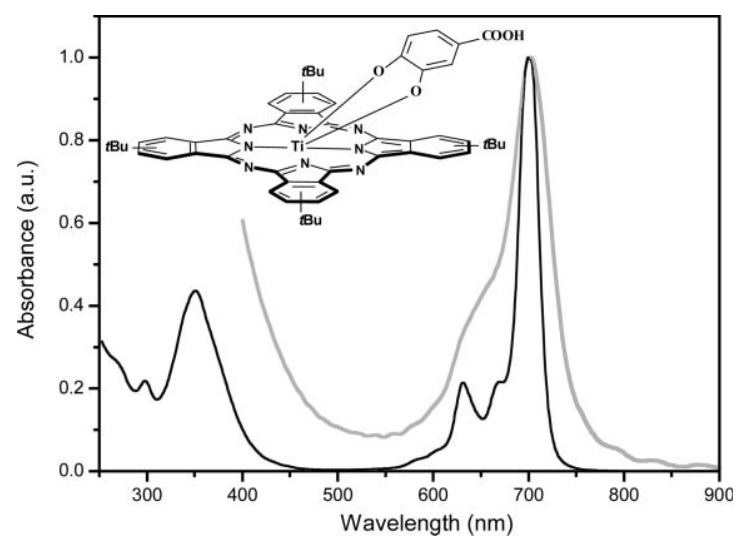

Fig. 1 Normalised absorption spectra of TiPc in dichloromethane (black) and adsorbed to a $\mathrm{TiO}_{2}$ film (grey). The inset shows the dye structure. 


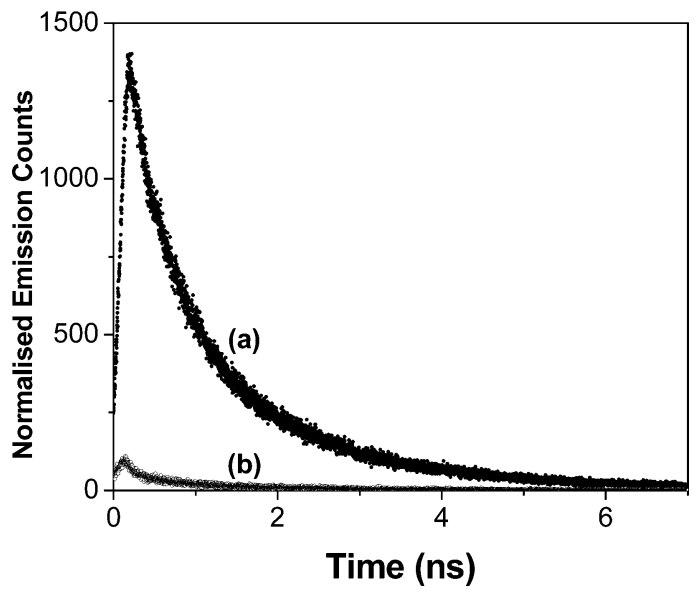

Fig. 2 Single photon counting fluorescence decays for (a) $\mathrm{TiPc} / \mathrm{ZrO}_{2}$ and (b) $\mathrm{TiPc} / \mathrm{TiO}_{2}$ films $\left(\lambda_{\mathrm{exc}}=405 \mathrm{~nm}, \lambda_{\mathrm{det}}=750 \mathrm{~nm}\right)$. Data have been normalized to the number of photons absorbed, allowing quantitative comparison of both the signal decay dynamics and magnitudes.

The slow recombination dynamics we report here are consistent with the expected axial attachment of the sensitiser dye to the $\mathrm{TiO}_{2}$, with the Pc macrocycle orientated parallel to the $\mathrm{TiO}_{2}$ surface. ZINDO/1 calculations assuming this orientation yield a dye $\mathrm{HOMO}-\mathrm{TiO}_{2}$ separation of $12 \AA$. In a previous study of a series of ruthenium bipyridyl dyes, we have reported a strong correlation between the recombination dynamics and the spatial separation of the dye HOMO orbital from the $\mathrm{TiO}_{2}$ surface. ${ }^{7,8}$ The $12 \AA$ separation $(\sim 10 \AA$ to the carboxylate group), calculated here is in good agreement with the slow $(0.01 \mathrm{~s})$ recombination dynamics we observed here.

Remarkably, the magnitude of the $\mathrm{TiPc}^{+} / \mathrm{TiO}_{2}\left(\mathrm{e}^{-}\right)$transient absorbance signal was strongly dependent upon excitation wavelength, with negligible signal amplitude for excitation wavelengths of $\geqslant 600 \mathrm{~nm}$, as shown in Fig. 3. This observation is strongly indicative of state selective electron injection, with efficient charge separation following Soret band excitation but only negligible charge separation following Q-band excitation. Further support for this assignment comes from the incident photon-to-electron conversion efficiency spectra (IPCE) for the $\mathrm{TiPc} / \mathrm{TiO}_{2}$ films in complete 'sandwich configuration' DSSC's, which shows efficient photocurrent generation in the UV/blue spectral region, but only very low red photocurrent generation as illustrated in Fig. 4. The

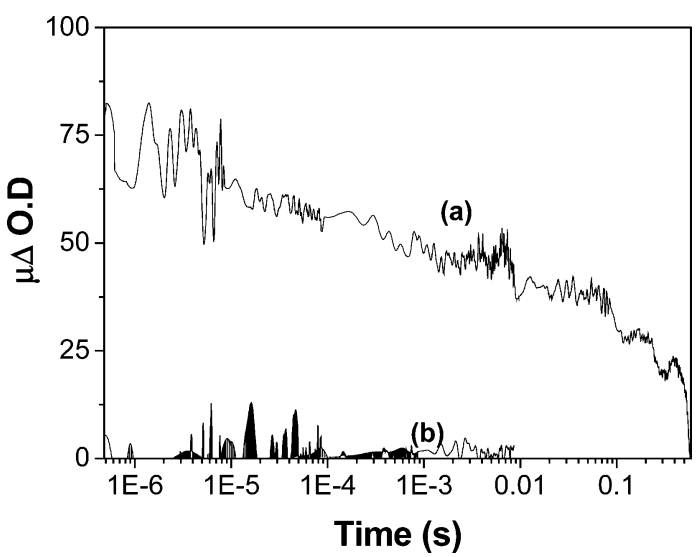

Fig. 3 Transient absorption data for $\mathrm{TiPc} / \mathrm{TiO}_{2}$ films after excitation at $450 \mathrm{~nm}$ (a) and $600 \mathrm{~nm}$ (b) $\left(\lambda_{\text {probe }}=850 \mathrm{~nm}\right)$.

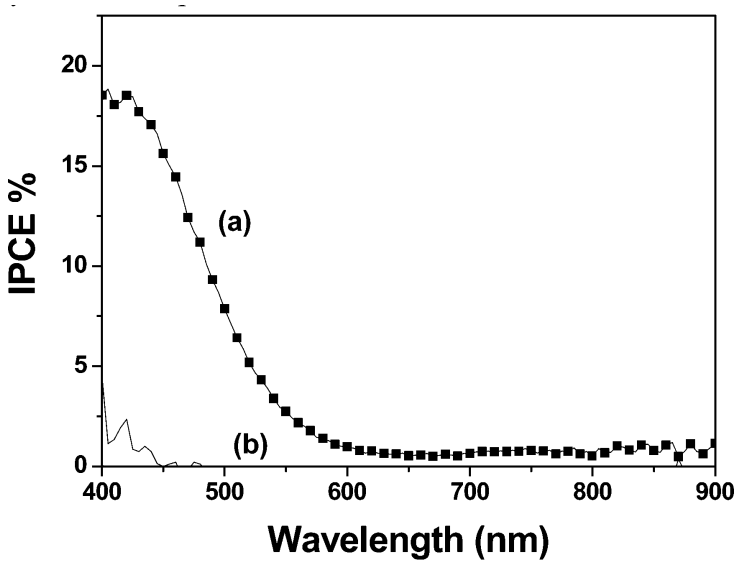

Fig. 4 IPCE spectra for $1 \mathrm{~cm}^{2}$ transparent DSSC's with (a) $\mathrm{TiPc} / \mathrm{TiO}_{2}$ photoelectrode and (b) control unsensitised $\mathrm{TiO}_{2}$ films. The electrolyte composition was $0.1 \mathrm{LiI}$ and $0.01 \mathrm{I}_{2}$ in propylene carbonate. $\mathrm{TiO}_{2}$ film thicknesses of $4 \mu \mathrm{m}$.

overall device energy conversion efficiency under AM1.5 irradiation was $0.2 \%$.

The observation of state selective electron injection for the TiPc/ $\mathrm{TiO}_{2}$ is indicative of efficient kinetic competition between electron injection from the $\mathrm{S}_{2}$ excited state of TiPc and internal conversion/ vibrational relaxation of this state to the lower lying $S_{1}$ state of this dye. The absence of efficient electron injection from the $S_{1}$ state is consistent with the low $E\left(\mathrm{D}^{*} / \mathrm{D}^{+}\right)$mid-point potential for this state $\left(\sim-0.8 \mathrm{~V}\right.$ versus $\mathrm{Ag} / \mathrm{AgCl}$ - close to the $\mathrm{TiO}_{2}$ conduction band edge) and the large dye LUMO-electrode surface separation determined from the semi-empirical calculations (see supplementary information $\dagger$ ). Clearly, optimisation of the photovoltaic energy conversion of DSSC's employing such dyes will require efficient electron injection from the $S_{1}$ state. Nevertheless we have shown for the first time the potential of TiPc's for the sensitisation of nanocrystalline $\mathrm{TiO}_{2}$ films. We have furthermore demonstrated that the combination of catechol axial attachment to the $\mathrm{TiO}_{2}$ surface and tert-butyl peripheral groups allows efficient sensitisation with no dye aggregation and without the use of any coadsorbents. It can be concluded that such TiPc dyes represent an attractive route to the development of efficient, red absorbing sensitiser dyes for dye sensitised photoelectrochemical solar cells.

The authors are grateful to the EPSRC and to the EU (Marie Curie Fellowship Contract No.HPMF-CT-2002-01744 for EP) for their financial support. This work was supported by CICYT (Spain), Comunidad de Madrid (Spain) and the European Union through grants BQU2002-04697, 07N/0030/2002 and HPRN-CT2000-00020, respectively.

\section{Notes and references}

1 B. O’Regan and M. Grätzel, Nature, 1991, 353, 737.

2 Md. K. Nazeeruddin, R. Humphry-Baker, M. Grätzel and B. A. Murrer, Chem. Commun., 1998, 719.

3 M. A. Loi, P. Denk, H. Hoppe, H. Neugebauer, C. Winder, D. Meissner, C. Brabec, S. Sariciftci, A. Gouloumis, P. Vazquez and T. J. Torres, Mater. Chem., 2003, 13, 700.

4 J. He, G. Benko, F. Korodi, T. Polivka, R. Lomoth, B. Akermark, L. Sun, A. Hafeldt and V. Sundstrom, J. Am. Chem. Soc., 2002, 124, 4923.

5 M. Barthel, D. Dini, S. Vagin and M. Hanack, Eur. J. Org. Chem., 2002, 3756.

6 M. Barthel and M. J. Hanack, Porphyrins Phthalocyanines, 2000, 4, 635.

7 J. N. Clifford, E. Palomares, Md. K. Nazeeruddin, M. Grätzel, J. Nelson,

X. Li, N. J. Long and J. R. Durrant, J. Am. Chem. Soc., 2004, 126, 5225.

8 N. Hirata, J.-J. Lagref, E. J. Palomares, J. R. Durrant, M. K. Nazeeruddin, M. Gratzel and D. Di Censo, Chem. Eur. J., 2004, 10, 595. 